\title{
Hankel Forms and embedding theorems in weighted dirichlet spaces
}

Article

Accepted Version

Aleman, A. and Perfekt, K.-M. (2011) Hankel Forms and embedding theorems in weighted dirichlet spaces.

International Mathematics Research Notices, 2012 (19). pp. 4435-4448. ISSN 1073-7928 doi:

https://doi.org/10.1093/imrn/rnr195 Available at https://centaur.reading.ac.uk/71747/

It is advisable to refer to the publisher's version if you intend to cite from the work. See Guidance on citing.

Published version at: http://dx.doi.org/10.1093/imrn/rnr195

To link to this article DOI: http://dx.doi.org/10.1093/imrn/rnr195

Publisher: Oxford University Press

All outputs in CentAUR are protected by Intellectual Property Rights law, including copyright law. Copyright and IPR is retained by the creators or other copyright holders. Terms and conditions for use of this material are defined in the End User Agreement.

\section{www.reading.ac.uk/centaur}

\section{CentAUR}

Central Archive at the University of Reading 
Reading's research outputs online 
A. Aleman and K-M. Perfekt. (0000) "Hankel Forms and Embedding Theorems in Weighted Dirichlet Spaces,"

International Mathematics Research Notices, Vol. 0000, Article ID rnn000, 12 pages.

doi:10.1093/imrn/rnn000

\title{
Hankel Forms and Embedding Theorems in Weighted Dirichlet Spaces
}

\author{
Alexandru Aleman and Karl-Mikael Perfekt \\ Department of Mathematics, Lund University, PO Box 118, S-221 00 Lund, Sweden
}

Correspondence to be sent to: perfekt@maths.lth.se

We show that for a fixed operator-valued analytic function $g$ the boundedness of the bilinear (Hankel-type) form $(f, h) \rightarrow \int_{\mathbb{D}} \operatorname{tr}\left(g^{\prime *} f h^{\prime}\right)\left(1-|z|^{2}\right)^{\alpha} \mathrm{d} A$, defined on appropriate cartesian products of dual weighted Dirichlet spaces of Schatten class-valued functions, is equivalent to corresponding Carleson embedding estimates.

\section{Introduction}

The characterization of bounded Hankel operators in terms of Carleson-type embeddings goes back to Fefferman's famous duality theorem for the Hardy space $H^{1}$ and has attracted a lot of attention during the past decades. The result can be stated in the following form: For a fixed analytic function $g$ in the unit disc $\mathbb{D}=\{z \in \mathbb{C}:|z|<1\}$ we have

$$
\sup _{\|f\|_{2},\|h\|_{2} \leq 1}\left|\lim _{r \rightarrow 1} \int_{\mathbb{D}_{r}} \overline{g^{\prime}}(f h)^{\prime}\left(1-|z|^{2}\right) \mathrm{d} A\right|^{2} \sim \sup _{\|f\|_{2} \leq 1} \int_{\mathbb{D}}\left|f g^{\prime}\right|^{2}\left(1-|z|^{2}\right) \mathrm{d} A,
$$

where $A$ denotes the usual area measure, $\|\cdot\|_{2}$ the norm on the Hardy space $H^{2}, \mathbb{D}_{r}=\{z \in \mathbb{C}:|z|<r\}$, and the functions $f, h$ above are polynomials. The bilinear form on the left hand side can easily be transformed into the usual Hankel form by simply replacing $h$ by $h^{*}(z)=\bar{h}(\bar{z})$, which, of course leaves the $H^{2}$-norm unchanged. Moreover, the quantity on the right hand side is the norm of the embedding operator from $H^{2}$ into $L^{2}\left(\mathbb{D},\left|g^{\prime}\right|^{2}\left(1-|z|^{2}\right) \mathrm{d} A\right)$, which is finite if and only if $g \in B M O A$.

The Hilbert space-valued version of (1) is obtained by considering operator-valued analytic functions $g$ and by replacing the modulus by the norm, and the products by scalar products. It is related to a number of important open questions in the area. In the infinite dimensional case, both the $B M O$-norm, and the Carleson embedding condition are not fully understood (see [10] and [9]). Further cases where the embedding conditions are more involved are given by the weighted Dirichlet spaces. Even for the classical unweighted Dirichlet space 
$\mathbf{D}^{2,0}(\mathbb{C})$ the corresponding estimate

$$
\sup _{\|f\|_{\mathbf{D}^{2,0},\|h\|_{\mathbf{D}^{2}, 0} \leq 1}}\left|\lim _{r \rightarrow 1} \int_{\mathbb{D}_{r}} \overline{g^{\prime}}(f h)^{\prime} \mathrm{d} A\right|^{2} \sim \sup _{\|f\|_{\mathbf{D}^{2}, 0} \leq 1} \int_{\mathbb{D}}\left|f g^{\prime}\right|^{2} \mathrm{~d} A
$$

has been proved only very recently in a somewhat indirect way by Arcozzi, Rochberg, Sawyer and Wick [2].

The present paper is concerned with a related class of Hankel forms that appear to be more tractable and in their turn have attracted some attention. They are obtained by replacing the term $(f h)^{\prime}$ above by $f h^{\prime}$. We shall work in the general context of Schatten class-valued weighted Dirichlet spaces which cover all situations discussed above. Given a fixed separable Hilbert space $\mathcal{H}$ we consider the usual Schatten classes $\mathcal{S}_{t}=\mathcal{S}_{t}(\mathcal{H}), 1<t<\infty$ and let $\mathbf{D}^{p, \beta}\left(\mathcal{S}_{t}\right), 1<p<\infty, \beta>-1$, be the space of $\mathcal{S}_{t}$-valued holomorphic functions $f$ in $\mathbb{D}$ with

$$
\|f\|_{\mathbf{D}^{p, \beta}\left(\mathcal{S}_{t}\right)}=\|f(0)\|_{\mathcal{S}_{t}}+\left(\int_{\mathbb{D}}\left\|f^{\prime}(z)\right\|_{\mathcal{S}_{t}}^{p}\left(1-|z|^{2}\right)^{\beta} \mathrm{d} A(z)\right)^{1 / p}<\infty
$$

It follows from results in [11] (see also Section 2 below) that the dual of $\mathbf{D}^{p, \beta}\left(\mathcal{S}_{p^{\prime}}\right)$ with respect to the $\mathbf{D}^{2, \alpha_{-}}$ pairing can be identified with $\mathbf{D}^{q, \gamma}\left(\mathcal{S}_{q^{\prime}}\right)$, in the sense that every continuous linear functional $\ell$ on $\mathbf{D}^{p, \beta}\left(\mathcal{S}_{p^{\prime}}\right)$ can be written as

$$
\ell(f)=\operatorname{tr}\left(f(0) g(0)^{*}\right)+\int_{\mathbb{D}} \operatorname{tr}\left(f^{\prime}(z) g^{\prime}(z)^{*}\right)\left(1-|z|^{2}\right)^{\alpha} \mathrm{d} A(z),
$$

for some unique $g \in \mathbf{D}^{q, \gamma}\left(\mathcal{S}_{q^{\prime}}\right)$ with $\|\ell\| \sim\|g\|$. Here $\operatorname{tr}(T)$ denotes the trace of the operator $T$, and $1<$ $p, p^{\prime}, q, q^{\prime}<\infty, \alpha \geq 0$, and $\beta, \gamma>-1$ satisfy the relations $\frac{1}{p}+\frac{1}{q}=1, \frac{1}{p^{\prime}}+\frac{1}{q^{\prime}}=1$, and $\frac{\beta}{p}+\frac{\gamma}{q}=\alpha$.

We are interested in the boundedness of the Hankel form defined on $\mathbf{D}^{p, \beta}\left(\mathcal{S}_{p^{\prime}}\right) \times \mathbf{D}^{q, \gamma}\left(\mathcal{S}_{q^{\prime}}\right)$ by

$$
(f, h) \rightarrow \int_{\mathbb{D}} \operatorname{tr}\left(g^{\prime}(z)^{*} f(z) h^{\prime}(\bar{z})^{*}\right)\left(1-|z|^{2}\right)^{\alpha} \mathrm{d} A(z),
$$

where $g$ is a fixed analytic operator-valued function in $\mathbb{D}$. Let $B(x, y)=\frac{\Gamma(x) \Gamma(y)}{\Gamma(x+y)}$ denote the beta function. Note that in the scalar case $\mathcal{S}_{p^{\prime}}=\mathcal{H}=\mathbb{C}$, with respect to the standard orthonormal basis of $\mathbf{D}^{2, \alpha}(\mathbb{C})$ given by $e_{0}=1$,

$$
e_{n}(z)=\frac{1}{\sqrt{\pi} n} B(\alpha+1, n)^{-1 / 2} z^{n}, \quad n \geq 1
$$

our form corresponds to the matrix $\left(A_{i j}\right)$, with $A_{i 0}=0, A_{0 j}=\pi j B(\alpha+1, j)^{-\frac{1}{2}} B(\alpha+1, i+j) \overline{\hat{g}(j)}$, and

$$
A_{i j}=\frac{i+j}{i} B(\alpha+1, i)^{-\frac{1}{2}} B(\alpha+1, j)^{-\frac{1}{2}} B(\alpha+1, i+j) \overline{\hat{g}(i+j)}
$$

for $i, j \geq 1$, where $\hat{g}(n)$ denotes the $n$-th Taylor coefficient of $g$. By Stirling's formula,

$$
A_{i j} \sim B_{i j}=i^{\frac{\alpha-1}{2}} j^{\frac{\alpha+1}{2}}(i+j)^{-\alpha} \overline{\hat{g}(i+j)} .
$$


Schatten class-membership of matrices of type $\left(B_{i j}\right)$ has been considered in the vector-valued case by Peller in [11] for $\alpha>0$ when $p \leq 2$ and $\alpha>\frac{1}{2}-\frac{1}{p}$ when $p>2$. In the scalar-valued setting for $p=2$, the boundedness of the form (2) has been characterized by Rochberg and $\mathrm{Wu}$ [13] in terms of the corresponding embedding condition, that is, they have obtained an analogue of (1) in this context.

The main result of this paper extends the estimates of Rochberg and Wu to the general context considered here. In Section 3 we prove that for an analytic operator-valued function $g$ in $\mathbb{D}$

$$
\begin{aligned}
\sup _{\|f\|_{\mathbf{D}^{p, \beta},\|h\|_{\mathbf{D}^{q, \gamma}} \leq 1}} \mid \lim _{r \rightarrow 1} \int_{\mathbb{D}_{r}} \operatorname{tr}\left(g^{\prime}(z)^{*} f(z) h^{\prime}(\bar{z})^{*}\right)(1 & \left.-|z|^{2}\right)^{\alpha} \mathrm{d} A(z) \mid \\
& \sim \sup _{\|f\|_{\mathbf{D}^{p, \beta}} \leq 1}\left(\int_{\mathbb{D}}\left\|g^{\prime}(z)^{*} f(z)\right\|_{\mathcal{S}_{p^{\prime}}}^{p}\left(1-|z|^{2}\right)^{\beta} \mathrm{d} A(z)\right)^{1 / p} .
\end{aligned}
$$

When $\beta>p-1$, the space $\mathbf{D}^{p, \beta}\left(\mathcal{S}_{p^{\prime}}\right)$ is a weighted Bergman space and we show that the quantities in (3) are bounded if and only if $g$ belongs to the Bloch space, that is, if and only if $\left(1-|z|^{2}\right)\left\|g^{\prime}(z)\right\|$ is bounded in $\mathbb{D}$. In this case we can replace $g^{\prime}(z)^{*}$ by $g^{\prime}(z)$ on the right hand side of (3). A more surprising fact is that in general this is no longer possible when $\beta \leq p-1$. When $\operatorname{dim} \mathcal{H}=\infty, p=p^{\prime}=2$, and $\beta \leq 1$, we construct examples of rank-one operator-valued analytic functions $g$ in $\mathbb{D}$ such that the quantities in (3) are infinite, but

$$
\sup _{\|f\|_{\mathbf{D}^{2, \beta}} \leq 1}\left(\int_{\mathbb{D}}\left\|g^{\prime}(z) f(z)\right\|_{\mathcal{S}_{2}}^{2}\left(1-|z|^{2}\right)^{\beta} \mathrm{d} A(z)\right)^{1 / 2}<\infty .
$$

In the dyadic case similar examples have been constructed in [4]. We are grateful to Sandra Pott for helpful discussions on this subject.

Our estimate (3) can be interpreted in terms of duality. More precisely, it shows that the embedding condition characterizes the dual of the weak product space $\partial^{-1}\left(\mathbf{D}^{p, \beta}\left(\mathcal{S}_{p^{\prime}}\right) \odot \partial \mathbf{D}^{q, \gamma}\left(\mathcal{S}_{q^{\prime}}\right)\right)$, consisting of analytic functions $F: \mathbb{D} \rightarrow \mathcal{S}_{1}$ with derivatives of the form

$$
F^{\prime}=\sum_{n=0}^{\infty} f_{n} h_{n}^{\prime}
$$

where $f_{n} \in \mathbf{D}^{p, \beta}\left(\mathcal{S}_{p^{\prime}}\right), h_{n} \in \mathbf{D}^{q, \gamma}\left(\mathcal{S}_{q^{\prime}}\right)$, and $\sum\left\|f_{n}\right\|_{\mathbf{D}^{p, \beta}}\left\|h_{n}\right\|_{\mathbf{D}^{q, \gamma}}<\infty$. The norm is given by

$$
\|F\|=\|F(0)\|_{\mathcal{S}_{1}}+\inf \sum_{n}\left\|f_{n}\right\|_{\mathbf{D}^{p, \beta}}\left\|h_{n}\right\|_{\mathbf{D}^{q, \gamma}},
$$

where the infimum is taken over all possible representations. With this point of view it is natural to compare (2) and the Hankel form

$$
(f, h) \rightarrow \int_{\mathbb{D}} \operatorname{tr}\left(g^{\prime}(z)^{*}\left(f(z) h(\bar{z})^{*}\right)^{\prime}\right)\left(1-|z|^{2}\right)^{\alpha} \mathrm{d} A(z),
$$

which, when bounded, corresponds to a continuous linear functional on $\mathbf{D}^{p, \beta}\left(\mathcal{S}_{p^{\prime}}\right) \odot \mathbf{D}^{q, \gamma}\left(\mathcal{S}_{q^{\prime}}\right)$. In the case when 
$\beta>p-1$ and $\gamma>q-1$, it is not difficult to verify that the two forms are equivalent. In the finite dimensional case this continues to hold true for the Hardy space case $\mathbf{D}^{p, \beta}\left(\mathcal{S}_{p^{\prime}}\right)=\mathbf{D}^{q, \gamma}\left(\mathcal{S}_{q^{\prime}}\right)=\mathbf{D}^{2,1}\left(\mathcal{S}_{2}\right)$ (see Aleksandrov and Peller [1]), and also for the Dirichlet space $\mathbf{D}^{2,0}\left(\mathcal{S}_{2}\right)$-case, but this latter statement is considerably more difficult to prove (see [2]). However, if $\mathcal{H}$ has infinite dimension it follows from the results of Davidson and Paulsen in [5] that in the $\mathbf{D}^{2,1}\left(\mathcal{S}_{2}\right)$-case these two forms are not equivalent (see also Pisier [12]).

\section{Preliminaries}

We begin with a brief discussion of duality for the weighted Dirichlet spaces considered in this paper. Given a Banach space $X$ and a finite measure $\mu$ on $\mathbb{D}$, we denote by $L_{X}^{p}(\mu), 1 \leq p<\infty$, the corresponding $L^{p}$-space of Bochner integrable $X$-valued functions, with the usual norm

$$
\|f\|_{L^{p}}=\left(\int_{\mathbb{D}}\|f\|_{X}^{p} \mathrm{~d} \mu\right)^{1 / p}<\infty
$$

We assume that $X^{*}$ is separable, which ensures that $\left(L^{p}\right)^{*} \simeq L^{q}$ with the usual pairing (see for example [6]). Let $\mathrm{d} \mu_{\alpha}=\left(1-|z|^{2}\right)^{\alpha} \mathrm{d} A, \alpha>-1$. We consider the Bergman space $L_{a}^{p, \alpha}(X)$, which is the closed subspace of $L_{X}^{p}\left(\mu_{\alpha}\right)$ consisting of analytic functions in $\mathbb{D}$. We shall use the following standard result, which is usually stated for scalar functions but continues to hold in the vector-valued case with the same proof.

Proposition 2.1 ([7]). Let $-1<\alpha, \beta<\infty$ and $1 \leq p<\infty$. If $\operatorname{dim} X \geq 1$, then

$$
Q_{\alpha} f(z)=\int_{\mathbb{D}} \frac{\left(1-|w|^{2}\right)^{\alpha}}{|1-\bar{w} z|^{\alpha+2}} f(w) \mathrm{d} A(w)
$$

is a bounded operator on $L_{X}^{p}\left(\mu_{\beta}\right)$ if and only if $\beta+1<(\alpha+1) p$. In particular,

$$
P_{\alpha} f(z)=\frac{\alpha+1}{\pi} \int_{\mathbb{D}} \frac{\left(1-|w|^{2}\right)^{\alpha}}{(1-\bar{w} z)^{\alpha+2}} f(w) \mathrm{d} A(w)
$$

boundedly projects $L_{X}^{p}\left(\mu_{\beta}\right)$ onto the space $L_{a}^{p, \beta}(X)$ if $\beta+1<(\alpha+1) p$.

This result has a number of applications, some of which are well known duality results. These are actually special cases of the description of the dual of a vector-valued Besov space (see [11]). We have that the dual of the Bergman space $L_{a}^{p, \beta}(X)$ can be identified with $L_{a}^{q, \gamma}\left(X^{*}\right)$ via the pairing

$$
\langle f, g\rangle=\int_{\mathbb{D}}\langle f(z), g(z)\rangle\left(1-|z|^{2}\right)^{\alpha} \mathrm{d} A(z)
$$

where $\alpha>-1, \frac{1}{p}+\frac{1}{q}=1$, and $\frac{\beta}{p}+\frac{\gamma}{q}=\alpha$ (see also [3]). For further reference, the following proposition states the corresponding result for the Dirichlet space $\mathbf{D}^{p, \beta}(X), 1<p<\infty, \beta>-1$, the space of $X$-valued holomorphic 
functions $f$ in $\mathbb{D}$ with

$$
\|f\|_{\mathbf{D}^{p, \beta}(X)}=\|f(0)\|_{X}+\left(\int_{\mathbb{D}}\left\|f^{\prime}(z)\right\|_{X}^{p}\left(1-|z|^{2}\right)^{\beta} \mathrm{d} A(z)\right)^{1 / p}<\infty .
$$

Proposition 2.2. Let $X$ be a Banach space with separable dual and let $1<p<\infty$. Suppose $\alpha, \beta, \gamma>-1$ are such that $\frac{\beta}{p}+\frac{\gamma}{q}=\alpha$, where $\frac{1}{p}+\frac{1}{q}=1$. Then $\left(\mathbf{D}^{p, \beta}(X)\right)^{*} \simeq \mathbf{D}^{q, \gamma}\left(X^{*}\right)$ under the $\mathbf{D}^{2, \alpha}$-pairing, in the sense that for every continuous linear functional $\ell \in\left(\mathbf{D}^{p, \beta}\right)^{*}$ there exists a unique $g \in \mathbf{D}^{q, \gamma}$ such that

$$
\ell(f)=\langle f(0), g(0)\rangle+\int_{\mathbb{D}}\left\langle f^{\prime}(z), g^{\prime}(z)\right\rangle\left(1-|z|^{2}\right)^{\alpha} \mathrm{d} A(z), f \in \mathbf{D}^{p, \beta},
$$

where $\left\langle f^{\prime}(z), g^{\prime}(z)\right\rangle=g^{\prime}(z)\left(f^{\prime}(z)\right)$. Furthermore, every $g \in \mathbf{D}^{q, \gamma}$ induces a continuous linear functional on $\mathbf{D}^{p, \beta}$ via (4), and $\|\ell\| \sim\|g\|_{\mathbf{D}^{q, \gamma}}$.

When $X=\mathcal{S}_{p^{\prime}}$ we will use the pairing given by

$$
\operatorname{tr}\left(f(0) g(0)^{*}\right)+\int_{\mathbb{D}} \operatorname{tr}\left(f^{\prime}(z) g^{\prime}(z)^{*}\right)\left(1-|z|^{2}\right)^{\alpha} \mathrm{d} A(z)
$$

where $\operatorname{tr}(T)$ denotes the trace of the operator $T$. An immediate consequence of this and Stokes' theorem that will be used below is the fact that if $\beta>p-1$ then $\mathbf{D}^{p, \beta}(X)=L_{a}^{p, \beta-p}(X)$.

Another application of Proposition 2.1 is the following refinement of the first estimate given there.

Lemma 2.3. Let $-1<\alpha, \beta<\infty$ and $1<p<\infty$ be such that $\beta+1<(\alpha+1) p$. For $f \in L_{a}^{p, \beta}(X)$, let

$$
R_{\alpha} f(z)=\int_{\mathbb{D}} \frac{\left(1-|w|^{2}\right)^{\alpha}}{|1-\bar{w} z|^{\alpha+1}|z-w|}\|f(w)\|_{X} \mathrm{~d} A(w)
$$

Then

$$
\left\|R_{\alpha} f\right\|_{L^{p}\left(\mu_{\beta}\right)} \lesssim\|f\|_{L_{a}^{p, \beta}(X)} .
$$

Proof. For $z \in \mathbb{D}$, let $d_{z}$ denote the pseudohyperbolic disk with pseudohyperbolic center and radius $z$ and $1 / 2$, respectively,

$$
d_{z}=\left\{w \in \mathbb{D}:\left|\frac{z-w}{1-\bar{w} z}\right|<\frac{1}{2}\right\}
$$

We decompose $R_{\alpha}$ into two parts:

$$
R_{\alpha} f(z)=\underbrace{\int_{\mathbb{D} \backslash d_{z}} \frac{\left(1-|w|^{2}\right)^{\alpha}}{|1-\bar{w} z|^{\alpha+1}|z-w|}\|f(w)\|_{X} \mathrm{~d} A(w)}_{R_{\alpha}^{\prime} f(z)}+\underbrace{\int_{d_{z}} \frac{\left(1-|w|^{2}\right)^{\alpha}}{|1-\bar{w} z|^{\alpha+1}|z-w|}\|f(w)\|_{X} \mathrm{~d} A(w)}_{R_{\alpha}^{\prime \prime} f(z)} .
$$


By Proposition 2.1 we have that $\left\|R_{\alpha}^{\prime} f\right\|_{L^{p}} \lesssim\|f\|_{L^{p}}$.

To estimate the remaining term, we note that

$$
\frac{\left(1-|w|^{2}\right)^{\alpha}}{|1-\bar{w} z|^{\alpha+1}} \lesssim \frac{1}{1-|z|^{2}}, \quad w \in d_{z}
$$

Hence,

$$
\left|R_{\alpha}^{\prime \prime} f(z)\right| \lesssim \frac{1}{1-|z|^{2}} \sup _{d_{z}}\|f\|_{X} \int_{d_{z}} \frac{1}{|z-w|} \mathrm{d} A(w) \lesssim \sup _{d_{z}}\|f\|_{X}
$$

Letting $\widetilde{d}_{z}$ denote the pseudohyperbolic disk with center $z$ and radius $3 / 4$ we obtain, by subharmonicity, that

$$
\left|R_{\alpha}^{\prime \prime} f(z)\right| \lesssim \sup _{d_{z}}\|f\|_{X} \lesssim \frac{1}{\left(1-|z|^{2}\right)^{2}} \int_{\widetilde{d}_{z}}\|f(w)\|_{X} \mathrm{~d} A(w)
$$

This shows that $\left\|R_{\alpha}^{\prime \prime} f\right\|_{L^{p}} \lesssim\|f\|_{L^{p}}$, since the right-hand side is dominated by the Hardy-Littlewood maximal function of $\|f(\cdot)\|_{X}$.

\section{Main Results}

As pointed out in the introduction the main result of this paper is the following theorem.

Theorem 3.1. Let $1<p, p^{\prime}<\infty, \alpha \geq 0$, and $\beta>-1$. Let $q$ and $q^{\prime}$ satisfy $\frac{1}{p}+\frac{1}{q}=1$ and $\frac{1}{p^{\prime}}+\frac{1}{q^{\prime}}=1$, and let $\gamma>-1$ be defined by $\frac{\beta}{p}+\frac{\gamma}{q}=\alpha$. Then for every analytic $\mathcal{B}(\mathcal{H})$-valued function $g$ in $\mathbb{D}$ we have

$$
\begin{aligned}
& \sup _{\|f\|_{\mathbf{D}^{p, \beta}\left(\mathcal{S}_{p^{\prime}}\right)},\|h\|_{\mathbf{D}^{q, \gamma}\left(\mathcal{S}_{q^{\prime}}\right)} \leq 1}\left|\lim _{r \rightarrow 1} \int_{\mathbb{D}_{r}} \operatorname{tr}\left(g^{\prime}(z)^{*} f(z) h^{\prime}(\bar{z})^{*}\right)\left(1-|z|^{2}\right)^{\alpha} \mathrm{d} A(z)\right| \\
& \sim \sup _{\|f\|_{\mathbf{D}^{p, \beta}\left(\mathcal{S}_{p^{\prime}}\right)} \leq 1}\left(\int_{\mathbb{D}}\left\|g^{\prime}(z)^{*} f(z)\right\|_{\mathcal{S}_{p^{\prime}}}^{p}\left(1-|z|^{2}\right)^{\beta} \mathrm{d} A(z)\right)^{1 / p},
\end{aligned}
$$

where $\mathbb{D}_{r}=\{z \in \mathbb{C}:|z|<r\}$ and the above suprema are computed for polynomials $f, h$.

The proof relies on the following simple observation.

Lemma 3.2. Suppose that $X$ is a Banach space and that $u: \overline{\mathbb{D}} \rightarrow X$ is continuous on $\overline{\mathbb{D}}$ and twice continuously differentiable in $\mathbb{D}$ with integrable Laplacian $\Delta u$. Then for any $w \in \mathbb{D}$ we have

$$
\partial u(w)=\frac{1}{2 \pi} \int_{\partial \mathbb{D}} \frac{\bar{\xi}}{(1-\bar{\xi} w)^{2}} u(\xi) \mathrm{d} s+\frac{1}{\pi} \int_{\mathbb{D}} \frac{1-|\xi|^{2}}{(1-\bar{\xi} w)(w-\xi)} \Delta u(\xi) \mathrm{d} A(\xi)
$$

where $\mathrm{d} s=\mathrm{d} s(\xi)$ is the length measure and $\partial$ is the Wirtinger operator $\partial=\frac{1}{2}\left(\partial_{x}-i \partial_{y}\right), w=x+i y$.

Proof. The statement follows from differentiation of the Green potential formula (see Corollary 3.8 in [7])

$$
u(w)=\frac{1}{2 \pi} \int_{\partial \mathbb{D}} \frac{1-|w|^{2}}{|\xi-w|^{2}} u(\xi) \mathrm{d} s(\xi)+\frac{2}{\pi} \int_{\mathbb{D}} \log \left|\frac{\xi-w}{1-\bar{\xi} w}\right| \Delta u(\xi) \mathrm{d} A(\xi)
$$


Proof of Theorem 3.1. Standard approximation arguments show that both quantities may in fact be computed over all $f$ and $h$ analytic on the closed disc $\overline{\mathbb{D}}$. Since the quantity on the left is smaller than the one on the right hand side, we can assume without loss of generality that the left hand side is finite, call its value $F_{g}$. In this case we can easily verify that $g$ belongs to the Bloch space. Indeed, let $f(z)=\frac{S}{(1-\bar{w} z)^{a}}, S \in \mathcal{S}_{p^{\prime}}$ and $h^{\prime}(\bar{z})^{*}=\frac{T}{(1-\bar{w} z)^{b}}, T \in \mathcal{S}_{q^{\prime}}$, where $a$ and $b$ are chosen so that $a+b=\alpha+2, a+1>\frac{\beta+2}{p}$, and $b>\frac{\gamma+2}{q}$. With these choices computations show that

$$
\|g\|_{\text {Bloch }}=\sup _{w \in \mathbb{D}}\left(1-|w|^{2}\right)\left\|g^{\prime}(w)\right\|_{\mathcal{B}(\mathcal{H})} \lesssim F_{g}=\sup _{\|f\|,\|h\| \leq 1}\left|\lim _{r \rightarrow 1^{-}} \int_{\mathbb{D}_{r}} \operatorname{tr}\left(g^{\prime}(z)^{*} f(z) h^{\prime}(\bar{z})^{*}\right)\left(1-|z|^{2}\right)^{\alpha} \mathrm{d} A(z)\right| .
$$

Let $f$ be an $\mathcal{S}_{p^{\prime}}$-valued holomorphic polynomial. For $\frac{1}{2}<\rho<1$ and $z \in \mathbb{D}$ consider the function

$$
u_{z}^{\rho}(w)=-\frac{f(w)^{*}}{\pi(1-\rho \bar{w} z)^{\alpha}} \int_{\mathbb{D}} \frac{\left(1-|\eta|^{2}\right)^{\alpha} g^{\prime}(\rho \eta)}{\overline{\eta-w}} \mathrm{~d} A(\eta)
$$

It is continuous in $\overline{\mathbb{D}}$ and satisfies (see Theorem 1.2.2. in [8])

$$
\partial_{w} u_{z}^{\rho}(w)=\frac{\left(1-|w|^{2}\right)^{\alpha}}{(1-\rho \bar{w} z)^{\alpha}} f(w)^{*} g^{\prime}(\rho w), \quad w \in \mathbb{D}
$$

If we apply Lemma 3.2 to $u_{z}^{\rho}$ with $w=\rho z$ the result is

$$
f(\rho z)^{*} g^{\prime}\left(\rho^{2} z\right)=\frac{1}{2 \pi} \underbrace{\int_{\partial \mathbb{D}} \frac{\bar{\xi}}{(1-\rho \bar{\xi} z)^{2}} u_{z}^{\rho}(\xi) \mathrm{d} s(\xi)}_{I_{1}(\rho, z)}+\frac{1}{\pi} \underbrace{\int_{\mathbb{D}}^{\frac{1-\rho}{(1-\rho \bar{\xi} z)(\rho z-\xi)} \Delta_{\xi}} u_{z}^{\rho}(\xi) \mathrm{d} A(\xi)}_{I_{2}(\rho, z)} .
$$

To prove the theorem we will estimate the norms of these two terms in $L_{\mathcal{S}_{p^{\prime}}}^{p}\left(\left(1-|z|^{2}\right)^{\beta} \mathrm{d} A(z)\right)$.

We proceed by duality in order to accomplish this for the term $I_{1}$. Let $H$ be a polynomial, not necessarily holomorphic, and recall that such polynomials are dense in $L_{\mathcal{S}_{q^{\prime}}}^{q}\left(\left(1-|z|^{2}\right)^{\gamma} \mathrm{d} A(z)\right)$. Noticing that $-\frac{1}{\eta-\xi}=\frac{\xi}{1-\bar{\eta} \xi}$ for $\xi \in \partial \mathbb{D}$, we obtain

$$
\begin{gathered}
\pi \int_{\mathbb{D}} \operatorname{tr}\left(I_{1}(\rho, z) H(z)\right)\left(1-|z|^{2}\right)^{\alpha} \mathrm{d} A(z)=\operatorname{tr}\left(\int_{\mathbb{D}}\left(1-|\eta|^{2}\right)^{\alpha} \int_{\partial \mathbb{D}} \frac{f(\xi)^{*} g^{\prime}(\rho \eta) h^{\prime}(\rho \bar{\xi})}{1-\bar{\eta} \xi} \mathrm{d} s(\xi) \mathrm{d} A(\eta)\right) \\
=2 \pi \operatorname{tr}\left(\int_{\mathbb{D}}\left(1-|\eta|^{2}\right)^{\alpha} f(\eta)^{*} g^{\prime}(\rho \eta) h^{\prime}(\rho \bar{\eta}) \mathrm{d} A(\eta)\right)=2 \pi \int_{\mathbb{D}} \frac{\operatorname{tr}\left(f(\eta) h^{\prime}(\rho \bar{\eta})^{*} g^{\prime}(\rho \eta)^{*}\right)}{}\left(1-|\eta|^{2}\right)^{\alpha} \mathrm{d} A(\eta),
\end{gathered}
$$

where $h(\xi)$ is given by $h(0)=0$ and

$$
h^{\prime}(\xi)=\int_{\mathbb{D}} \frac{H(z)\left(1-|z|^{2}\right)^{\alpha}}{(1-\xi z)^{2+\alpha}} \mathrm{d} A(z), \quad \xi \in \mathbb{D}
$$


Note that $h$ is analytic in $\overline{\mathbb{D}}$ and that $\|h\|_{\mathbf{D}^{q, \gamma}} \lesssim\|H\|_{L^{q}}$, by Proposition 2.1. A computation with power series and polar coordinates shows that

$$
\int_{\mathbb{D}} \overline{\operatorname{tr}\left(f(\eta) h^{\prime}(\rho \bar{\eta})^{*} g^{\prime}(\rho \eta)^{*}\right)}\left(1-|\eta|^{2}\right)^{\alpha} \mathrm{d} A(\eta)=\lim _{r \rightarrow 1^{-}} \int_{\mathbb{D}_{r}} \overline{\operatorname{tr}\left(f(\rho \eta) h^{\prime}\left(\rho^{2} \bar{\eta}\right)^{*} g^{\prime}(\eta)^{*}\right)}\left(1-|\eta|^{2}\right)^{\alpha} \mathrm{d} A(\eta) .
$$

In view of the duality between $L^{p}$ and $L^{q}$ we may finally conclude that

$$
\varliminf_{\rho \rightarrow 1}\left\|I_{1}(\rho, \cdot)\right\|_{L^{p}} \lesssim F_{g}\|f\|_{\mathbf{D}^{p, \beta}} .
$$

To deal with the second term $I_{2}$ we begin by calculating

$$
\Delta_{\xi} u_{z}^{\rho}(\xi)=4 \frac{\left(1-|\xi|^{2}\right)^{\alpha}}{(1-\rho \bar{\xi} z)^{\alpha}} f^{\prime}(\xi)^{*} g^{\prime}(\rho \xi)+4 \alpha \frac{\left(1-|\xi|^{2}\right)^{\alpha-1}(\rho z-\xi)}{(1-\rho \bar{\xi} z)^{\alpha+1}} f(\xi)^{*} g^{\prime}(\rho \xi)
$$

Hence we may further decompose $I_{2}$,

$$
I_{2}(\rho, z)=4 \underbrace{\int_{\mathbb{D}} \frac{\left(1-|\xi|^{2}\right)^{\alpha+1}}{(1-\rho \bar{\xi} z)^{\alpha+1}(\rho z-\xi)} f^{\prime}(\xi)^{*} g^{\prime}(\rho \xi) \mathrm{d} A(\xi)}_{I_{3}(\rho, z)}+4 \alpha \underbrace{\int_{\mathbb{D}} \frac{\left(1-|\xi|^{2}\right)^{\alpha}}{(1-\rho \bar{\xi} z)^{\alpha+2}} f(\xi)^{*} g^{\prime}(\rho \xi) \mathrm{d} A(\xi)}_{I_{4}(\rho, z)} .
$$

We have

$$
\left\|I_{3}(\rho, z)\right\|_{\mathcal{S}_{p^{\prime}}} \lesssim\|g\|_{\text {Bloch }} \int_{\mathbb{D}} \frac{\left(1-|\xi|^{2}\right)^{\alpha}}{|1-\rho \bar{\xi} z|^{\alpha+1}|\rho z-\xi|}\left\|f^{\prime}(\xi)\right\|_{\mathcal{S}_{p^{\prime}}} \mathrm{d} A(\xi)
$$

Since $\|g\|_{\text {Bloch }} \lesssim F_{g}$ it follows from Lemma 2.3 that

$$
\varliminf_{\rho \rightarrow 1}\left\|I_{3}(\rho, \cdot)\right\|_{L^{p}} \lesssim F_{g}\|f\|_{\mathbf{D}^{p, \beta}} .
$$

For the final term $I_{4}$ we again use duality. With $H$ and $h$ as earlier, a computation similar to the one corresponding to the term $I_{1}$ shows that

$$
\int_{\mathbb{D}} \operatorname{tr}\left(I_{4}(\rho, z) H(z)\right)\left(1-|z|^{2}\right)^{\alpha} \mathrm{d} A(z)=\lim _{r \rightarrow 1} \int_{\mathbb{D}_{r}} \overline{\operatorname{tr}\left(f(\rho \xi) h^{\prime}\left(\rho^{2} \bar{\xi}\right)^{*} g^{\prime}(\xi)^{*}\right)}\left(1-|\xi|^{2}\right)^{\alpha} \mathrm{d} A(\xi) .
$$

Therefore we obtain

$$
\varliminf_{\rho \rightarrow 1}\left\|I_{4}(\rho, \cdot)\right\|_{L^{p}} \lesssim F_{g}\|f\|_{\mathbf{D}^{p, \beta}} .
$$

Since $f(\rho z)^{*} g^{\prime}\left(\rho^{2} z\right)$ is a linear combination of $I_{1}(\rho, z), I_{2}(\rho, z)$, and $I_{4}(\rho, z)$, applying Fatou's lemma together with the inequalities (5), (6), and (7) gives

$$
\left\|g^{\prime *} f\right\|_{L^{p}}=\left\|f^{*} g^{\prime}\right\|_{L^{p}} \lesssim F_{g}\|f\|_{\mathbf{D}^{p, \beta}} .
$$


This concludes the proof.

The embedding condition in Theorem 3.1,

$$
\sup _{\|f\|_{\mathbf{D}^{p, \beta}\left(\mathcal{S}_{p^{\prime}}\right)} \leq 1} \int_{\mathbb{D}}\left\|g^{\prime}(z)^{*} f(z)\right\|_{\mathcal{S}_{p^{\prime}}}^{p}\left(1-|z|^{2}\right)^{\beta} \mathrm{d} A(z)<\infty
$$

is easily understood when $\beta>p-1$, i.e. when $\mathbf{D}^{p, \beta}\left(\mathcal{S}_{p^{\prime}}\right)$ is a weighted Bergman space, but, as pointed out in the introduction, for the remaining cases this condition is much more mysterious, as the proposition below indicates.

\section{Proposition 3.3.}

(i) If $\beta>p-1$ the quantities in Theorem 3.1 are finite if and only if $g$ belongs to the Bloch space.

(ii) If $\beta \leq 1$ and $\operatorname{dim} \mathcal{H}=\infty$ then there exists an analytic operator-valued function $g$ in $\mathbb{D}$ such that $g(z)$ has rank one for all $z \in \mathbb{D}$,

$$
\sup _{\|f\|_{\mathbf{D}^{2, \beta}\left(\mathcal{S}_{2}\right)} \leq 1} \int_{\mathbb{D}}\left\|g^{\prime}(z) f(z)\right\|_{\mathcal{S}_{2}}^{2}\left(1-|z|^{2}\right)^{\beta} \mathrm{d} A(z)<\infty
$$

but there exist rank-one operators $f_{0} \in \mathcal{S}_{2}$ such that

$$
\int_{\mathbb{D}}\left\|g^{\prime}(z)^{*} f_{0}\right\|_{\mathcal{S}_{2}}^{2}\left(1-|z|^{2}\right)^{\beta} \mathrm{d} A(z)=\infty
$$

Proof. (i) According to the proof of Theorem 3.1 the necessity holds true for any $\beta>-1$. Conversely, if $g$ belongs to the Bloch space and $\beta>p-1$, as pointed out in Section 2, we have that $\mathbf{D}^{p, \beta}\left(\mathcal{S}_{p^{\prime}}\right)=L_{a}^{p, \beta-p}\left(\mathcal{S}_{p^{\prime}}\right)$, so that

$$
\int_{\mathbb{D}}\left\|g^{\prime}(z)^{*} f(z)\right\|_{\mathcal{S}_{p^{\prime}}}^{p}\left(1-|z|^{2}\right)^{\beta} \mathrm{d} A(z) \lesssim \int_{\mathbb{D}}\|f(z)\|_{\mathcal{S}_{p^{\prime}}}^{p}\left(1-|z|^{2}\right)^{\beta-p} \mathrm{~d} A(z) \sim\|f\|_{\mathbf{D}^{p, \beta}\left(\mathcal{S}_{p^{\prime}}\right)}^{p}
$$

(ii) We may assume that $\mathcal{H}=H^{2}=\mathbf{D}^{2,0}(\mathbb{C})$ is the usual scalar-valued Hardy space. Furthermore, under this assumption, note that

$$
\sup _{\|f\|_{\mathbf{D}^{2, \beta}\left(\mathcal{S}_{2}\right)} \leq 1} \int_{\mathbb{D}}\left\|g^{\prime} f\right\|_{\mathcal{S}_{2}}^{2}\left(1-|z|^{2}\right)^{\beta} \mathrm{d} A(z)=\sup _{\|h\|_{\mathbf{D}^{2, \beta}\left(H^{2}\right)} \leq 1} \int_{\mathbb{D}}\left\|g^{\prime} h\right\|_{H^{2}}^{2}\left(1-|z|^{2}\right)^{\beta} \mathrm{d} A(z) .
$$

To see that the left quantity is less than the one on the right compute with a fixed orthonormal basis of $H^{2}$. For the converse inequality, consider functions $f$ such that $f(z)$ has rank one for every $z$.

Let $a_{k}=k^{\beta / 2}$ and define $u: \mathbb{D}^{2} \rightarrow \mathbb{C}$ by $u(z, w)=\sum_{k} \frac{a_{k}}{k+1} \bar{z}^{k+1} w^{k}$, and let $g: \mathbb{D} \rightarrow \mathcal{B}\left(H^{2}\right)$ be given by

$$
g(z) x \equiv\langle x, u(z, \cdot)\rangle_{H^{2}}, \quad x \in H^{2}
$$


That is, $g(z) x$ is always a constant function, so that $g(z)$ certainly has rank one. Note that $g^{\prime}(z) x \equiv$ $\left\langle x, \bar{\partial}_{z} u(z, \cdot)\right\rangle_{H^{2}}$ and

$$
g^{\prime}(z)^{*} x=x(0) \bar{\partial}_{z} u(z, \cdot)
$$

We claim that this $g$ has the desired properties. Indeed, given $h \in \mathbf{D}^{2, \beta}\left(H^{2}\right)$ we write $h(z, w)=(h(z))(w)=$ $\sum_{n, k} h_{n k} z^{n} w^{k}$ as a power series and compute

$$
\begin{aligned}
\int_{\mathbb{D}}\left\|g^{\prime} h\right\|_{H^{2}}^{2}\left(1-|z|^{2}\right)^{\beta} \mathrm{d} A(z)=\int_{\mathbb{D}}\left|\sum_{m=0}^{\infty}\left(\sum_{n+k=m} a_{k} h_{n k}\right) z^{m}\right|^{2}\left(1-|z|^{2}\right)^{\beta} \mathrm{d} A(z) \\
\sim \sum_{m=0}^{\infty} \frac{1}{(m+1)^{\beta+1}}\left|\sum_{n+k=m} a_{k} h_{n k}\right|^{2} \leq \sum_{m=0}^{\infty} \frac{1}{(m+1)^{\beta+1}} \sum_{k=0}^{m} a_{k}^{2} \sum_{n=0}^{m}\left|h_{n, m-n}\right|^{2}(n+1)^{1-\beta} \\
\quad \lesssim \sum_{m=0}^{\infty} \sum_{n=0}^{m}\left|h_{n, m-n}\right|^{2}(n+1)^{1-\beta} \sim\|h\|_{\mathbf{D}^{2, \beta}\left(H^{2}\right)}
\end{aligned}
$$

This shows that (8) holds. Note that the calculation above relies on the fact that $\sup _{m} \frac{1}{(m+1)^{\beta+1}} \sum_{k=0}^{m} a_{k}^{2}<\infty$.

To obtain (9) choose $0 \neq y, z \in H^{2}$ with $z(0) \neq 0$ and define $f_{0}$ by $f_{0} x=\langle x, y\rangle_{H^{2}} z, x \in H^{2}$. Then

$$
\begin{aligned}
\int_{\mathbb{D}}\left\|g^{\prime}(z)^{*} f_{0}\right\|_{\mathcal{S}_{2}}^{2}\left(1-|z|^{2}\right)^{\beta} \mathrm{d} A(z) & \sim \int_{\mathbb{D}}\left\|\bar{\partial}_{z} u(z, \cdot)\right\|_{H_{2}}^{2}\left(1-|z|^{2}\right)^{\beta} \mathrm{d} A(z) \\
& =\sum_{k} a_{k}^{2} \int_{\mathbb{D}}|z|^{2 k}\left(1-|z|^{2}\right)^{\beta} \mathrm{d} A(z) \sim \sum_{k} \frac{a_{k}^{2}}{(k+1)^{\beta+1}}=\infty
\end{aligned}
$$

as desired.

The main result has a reformulation in terms of duality which is given below.

Corollary 3.4. Under the assumptions of Theorem 3.1, define the space $\mathcal{X}$ as

$$
\mathcal{X}=\left\{g:\|g\|_{\mathcal{X}}<\infty\right\}
$$

where $g: \mathbb{D} \rightarrow \mathcal{B}(\mathcal{H})$ is analytic and $\|\cdot\|_{\mathcal{X}}$ is the corresponding norm

$$
\|g\|_{\mathcal{X}}=\|g(0)\|+\sup _{\|f\|_{\mathbf{D}^{p, \beta}\left(\mathcal{S}_{p^{\prime}}\right)} \leq 1}\left(\int_{\mathbb{D}}\left\|g^{\prime}(z)^{*} f(z)\right\|_{\mathcal{S}_{p^{\prime}}}^{p}\left(1-|z|^{2}\right)^{\beta} \mathrm{d} A(z)\right)^{1 / p}
$$

Then $\left(\partial^{-1}\left(\mathbf{D}^{p, \beta}\left(\mathcal{S}_{p^{\prime}}\right) \odot \partial \mathbf{D}^{q, \gamma}\left(\mathcal{S}_{q^{\prime}}\right)\right)^{*} \simeq \mathcal{X}\right.$ via the $\mathbf{D}^{2, \alpha}$-pairing, in the sense that for every bounded functional $\ell \in\left(\partial^{-1}\left(\mathbf{D}^{p, \beta} \odot \partial \mathbf{D}^{q, \gamma}\right)\right)^{*}$ there is a unique $g \in \mathcal{X}$ such that

$$
\ell(F)=\operatorname{tr}\left(F(0) g(0)^{*}\right)+\int_{\mathbb{D}} \operatorname{tr}\left(F^{\prime}(z) g^{\prime}(z)^{*}\right)\left(1-|z|^{2}\right)^{\alpha} \mathrm{d} A(z)
$$


for all $F \in \partial^{-1}\left(\mathbf{D}^{p, \beta} \odot \partial \mathbf{D}^{q, \gamma}\right)$. Furthermore, every $g \in \mathcal{X}$ induces a bounded linear functional via (10), and $\|\ell\| \sim\|g\|_{\mathcal{X}}$.

Proof. It is obvious that every $g \in \mathcal{X}$ induces a continuous linear functional $\ell$ which satisfies $\|\ell\| \lesssim\|g\|_{\mathcal{X}}$. Conversely, assume that $\ell \in\left(\partial^{-1}\left(\mathbf{D}^{p, \beta} \odot \partial \mathbf{D}^{q, \gamma}\right)\right)^{*}$. For $n \geq 0$, define $g_{n} \in \mathcal{B}(\mathcal{H})$ by

$$
\operatorname{tr}\left(T g_{n}^{*}\right)=\ell\left(z^{n} T\right), \quad T \in \mathcal{S}_{1},
$$

and let $g: \mathbb{D} \rightarrow \mathcal{B}(\mathcal{H})$ be given by

$$
g(z)=g_{0}+\frac{1}{\pi} \sum_{n=1}^{\infty} \frac{1}{n^{2} B(\alpha+1, n)} g_{n} z^{n},
$$

where $B$ is again the usual Beta function. A computation with power series and polar coordinates demonstrates that

$$
\ell(h)=\operatorname{tr}\left(h(0) g(0)^{*}\right)+\lim _{r \rightarrow 1^{-}} \int_{\mathbb{D}_{r}} \operatorname{tr}\left(h^{\prime}(z) g^{\prime}(z)^{*}\right)\left(1-|z|^{2}\right)^{\alpha} \mathrm{d} A(z)
$$

at least for $\mathcal{S}_{1}$-valued polynomials $h$. The boundedness of $\ell$ in conjunction with Theorem 3.1 now easily implies that $g \in \mathcal{X}$, so that (10) in fact holds. Choosing $F$ so that $F^{\prime}(z)=\frac{T}{(1-\bar{w} z)^{2+\alpha}}, T \in \mathcal{S}_{1}$, establishes the uniqueness of $g$. The remaining inequality $\|g\|_{\mathcal{X}} \lesssim\|\ell\|$ is a consequence of the closed graph theorem.

\section{References}

[1] Aleksandrov, A. B., Peller, V. V., Hankel operators and similarity to a contraction. Internat. Math. Res. Notices 1996, no. 6, 263-275.

[2] Arcozzi, N., Rochberg, R., Sawyer, E., Wick, B. D., Bilinear forms on the Dirichlet space. Anal. PDE 3 (2010), no. 1, 21-47

[3] Arregui J. L., Blasco O., Bergman and Bloch spaces of vector-valued functions, Math. Nachr. 261/262 (2003), 3-22.

[4] Blasco, O., Pott, S., Embeddings between operator-valued dyadic BMO spaces. Illinois J. Math. 52 (2008), no. $3,799-814$.

[5] Davidson, K. R., Paulsen, V. I., Polynomially bounded operators. J. Reine Angew. Math. 487 (1997), $153-170$.

[6] Diestel, J., Uhl, J. J., "Vector measures", Mathematical Surveys, 15, (Amer. Math. Soc., R.I., 1977).

[7] Hedenmalm, H., Korenblum, B., Zhu, K., "Theory of Bergman spaces", Graduate Texts in Mathematics, 199. Springer-Verlag, New York, 2000. 
[8] Hörmander, L., "An Introduction to Complex Analysis in Several Variables", D. Van Nostrand Co., Inc., Princeton, N.J.-Toronto, Ont.-London 1966.

[9] Nazarov, F., Treil, S., Volberg, A., Counterexample to the infinite-dimensional Carleson embedding theorem, C. R. Acad. Sci. Paris Sr. I Math. 325 (1997), no. 4, 383-388.

[10] Nazarov, F., Pisier, G., Treil, S., Volberg, A, Sharp estimates in vector Carleson imbedding theorem and for vector paraproducts, J. Reine Angew. Math. 542 (2002), 147-171.

[11] Peller, V. V., Vectorial Hankel operators, commutators and related operators of the Schatten-von Neumann class $\gamma_{p}$. Integral Equations Operator Theory 5 (1982), no. 2, 244-272.

[12] Pisier, G., Un opérateur polynomialement borné sur un Hilbert qui n'est pas semblable à une contraction, C. R. Acad. Sci. Paris Sr. I Math. 322 (1996), no. 6, 547-550.

[13] Rochberg, R., Wu, Z. J., A new characterization of Dirichlet type spaces and applications, Illinois J. Math. 37 (1993), no. 1, 101-122. 